Bull. Mater. Sci., Vol. 36, No. 6, November 2013, pp. 1073-1077. (C) Indian Academy of Sciences.

\title{
Intrinsic structure and friction properties of graphene and graphene oxide nanosheets studied by scanning probe microscopy
}

\author{
YAN-HUAI DING**, HU-MING REN, FEI-HU CHANG, PING ZHANG and YONG JIANG \\ Institute of Rheology Mechanics, Xiangtan University, Xiangtan, China
}

MS received 2 May 2012; revised 6 August 2012

\begin{abstract}
In this paper, atomic structure of single-layered graphene oxide (GO) and chemically reduced graphene oxide (CRGO) nanosheets was investigated using atomic force microscopy and scanning tunneling microscopy (AFM and STM). Furthermore, friction properties of the graphene and GO nanosheets were studied by frictional force microscopy (FFM). STM imaging provided direct evidence and the morphology was influenced by oxygen-containing groups and defects. The atomic scale structural disorder in a hexagonal two-dimensional network of carbon atoms changes the surface condition, which also caused the frictional property variations of the samples.
\end{abstract}

Keywords. Graphene oxide; graphene; AFM; STM; FFM; frictional property.

\section{Introduction}

Graphene, a single layer of graphite, has attracted great interest in recent years because of the unique physical, chemical and mechanical properties arising from its strictly 2-D structure. The unique properties hold great promise for potential applications in many technological fields such as nanoelectronics, sensors, nanocomposites, batteries, supercapacitors and hydrogen storage (Allen et al 2010; Dreyer et al 2010; Loh et al 2010). Since Geim and coworkers at Manchester University first isolated single-layer graphene from bulk graphite in small amounts by micromechanical cleavage, many methods have been developed for preparing graphene including chemical vapour deposition (CVD), epitaxial growth and chemical reduction of exfoliated graphite oxide (Shioyama 2001; Pan et al 2009; Park and Ruoff 2009). Of these routes, chemical method is considered to be an efficient approach to producing processable graphene sheets in bulk quantity at a relatively low cost.

In general, graphite oxide has been mainly prepared by the modified Hummer's methods. These methods involve oxidation of graphite powder in the presence of strong acids and oxidants. The $s p^{2}$-bonded carbon network in graphite is readily destroyed and some parts of the $\mathrm{C}$ atoms are bonded to hydroxyl groups and epoxide groups and edges of the layers are attached to carboxylic or carbonyl groups. GO is strongly hydrophilic and is easily exfoliated in water because, large amounts of oxygen containing groups existed on the basal or edge plane. Reduction of the graphene oxide using reductants such as hydrazine, dimethylhydrazine and $\mathrm{NaBH}_{4}$ caused the agglomeration and precipitation of CRGO (Stankovich et al 2006, 2007; Muszynski et al 2008; Wang et al 2008). Most studies suggested that the graphene

\footnotetext{
*Author for correspondence (yhding@xtu.edu.cn)
}

stemmed from reduction of graphene oxide which contained a significant amount of oxygen groups and a large number of defects (Gass et al 2008; Allen et al 2010). Because, the electronic, thermal and mechanical properties of graphene and GO are exceptionally sensitive to the atomic structure and it is critically important to get information about the functional groups and defects of graphene prepared by chemical methods.

Scanning probe microscopy is one of the most powerful modern research techniques that allows study of morphological features and local properties of the surface with high spatial resolution. In this paper, we used AFM and STM to probe the local atomic structure and morphology of single layer GO and graphene nanosheets under ambient conditions. Microtribological behaviours of single-layer graphene and GO sheets on mica surface were also tested by FFM.

\section{Experimental}

\subsection{Preparation of $G O$ and graphene}

Graphite oxide was prepared from natural graphite powder by a modified Hummers method (Ding et al 2010). In a typical procedure, $1.5 \mathrm{~g}$ graphite powders were first oxidized by reacting them in a mixture of $35 \mathrm{ml}$ concentrated nitric acid and $70 \mathrm{ml}$ concentrated sulfuric acid in an ice bath. And then, $9 \mathrm{~g}$ potassium permanganate was added to the container slowly (Notice: the reaction will get out of control if potassium permanganate was added quickly). After reaction at $65^{\circ} \mathrm{C}$ for $120 \mathrm{~h}, 100 \mathrm{ml}$ deionized water was added dropwise to the container carefully. Then the reaction was allowed to go on at $90{ }^{\circ} \mathrm{C}$ for $2 \mathrm{~h}$ to fully oxidize graphite into graphite oxide. The graphite oxide was washed with dilute hydrochloric acid and deionized water until $\mathrm{pH}$ of the washing solution increased to neutral $(\sim 7)$. Graphite oxide was dispersed in 
a mixture of deionized water and $N, N^{\prime}$-dimethylformamide (DMF) and exfoliated through ultrasonication for $1 \mathrm{~h}$. The final GO dispersion was reduced by hydrazine hydrate at $100{ }^{\circ} \mathrm{C}$ for $24 \mathrm{~h}$.

\subsection{Characterization}

Powder X-ray diffraction (XRD) was carried out using a Bruker D8 Advance X-ray diffractometer. AFM measurements for nanometer-scale morphology were taken with a Digital Instruments NS3D from Veeco in tapping mode using silicon tips with a resonance frequency of $125 \mathrm{kHz}$. STM measurements were performed in the constant current mode with mechanically prepared $\mathrm{Pt} / \mathrm{Ir}$ tips and $A(E)$ scanners. Microtribological behaviours of single-layer graphene and GO sheets on mica surface were also tested by FFM using a silicon RTESP tip. All STM and AFM experiments were carried out under the same test condition.

\section{Results and discussion}

XRD patterns of the natural graphite, GO and CRGO are recorded in figure 1. Agglomeration is caused easily in the process of preparation of GO and graphene sheets because of their higher surface energy. Compared with pristine graphite, stacking structure is still observed in GO and graphene powders and the layer-to-layer distance can be calculated by Bragg equation. The feature diffraction peak of exfoliated GO appears at $11.0^{\circ}$ with increased layer-to-layer distance of $0.80 \mathrm{~nm}$, which was identical with those reported in literature (Wang et al 2008). The expanded layer spacing was caused by intercalated hydroxyl, epoxy and carboxyl groups between layers. After the exfoliated GO is chemically reduced, the diffraction peak of CRGO is similar to that of graphite with significantly reduced intensity.

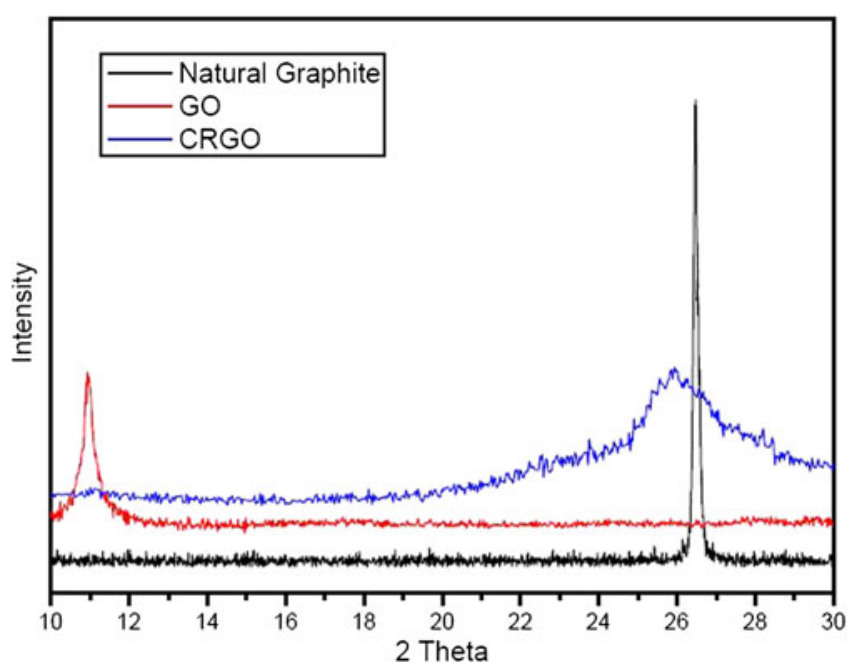

Figure 1. XRD patterns of natural graphite (black line), GO (red line) and CRGO (blue line) powders. GO and graphene showing expanded basal spacing.
This implies that most of the functional groups are removed and it should be noted that graphene sheets tend to form irreversible agglomerates or even re-stack to form graphite through strong $\pi-\pi$ stacking and Van der Waals interaction (Li and Kaner 2008).

For AFM and STM investigations, unreduced GO and CRGO dispersions were drop-cast onto freshly cleaved, atomically flat and highly oriented pyrolytic graphite (HOPG) substrates (ZYH grade, from SPI corporation) and then allowed to dry in air at room temperature. AFM was carried out in tapping mode with a super sharp tip to obtain high spatial resolution images under ambient conditions (relative humidity, $\sim 45 \%$ and temperature, $\sim 25{ }^{\circ} \mathrm{C}$ ). As shown in figure 2 , exfoliated GO are flat sheets with an average thickness of about $1.5 \mathrm{~nm}$. Thickness of CRGO sheets is about $1.0 \mathrm{~nm}$ with lateral dimension in the range of hundred nanometers to several microns. Roughness of GO and CRGO (in $1 \times 1 \mu \mathrm{m}$ region) are 0.367 and $0.153 \mathrm{~nm}$,
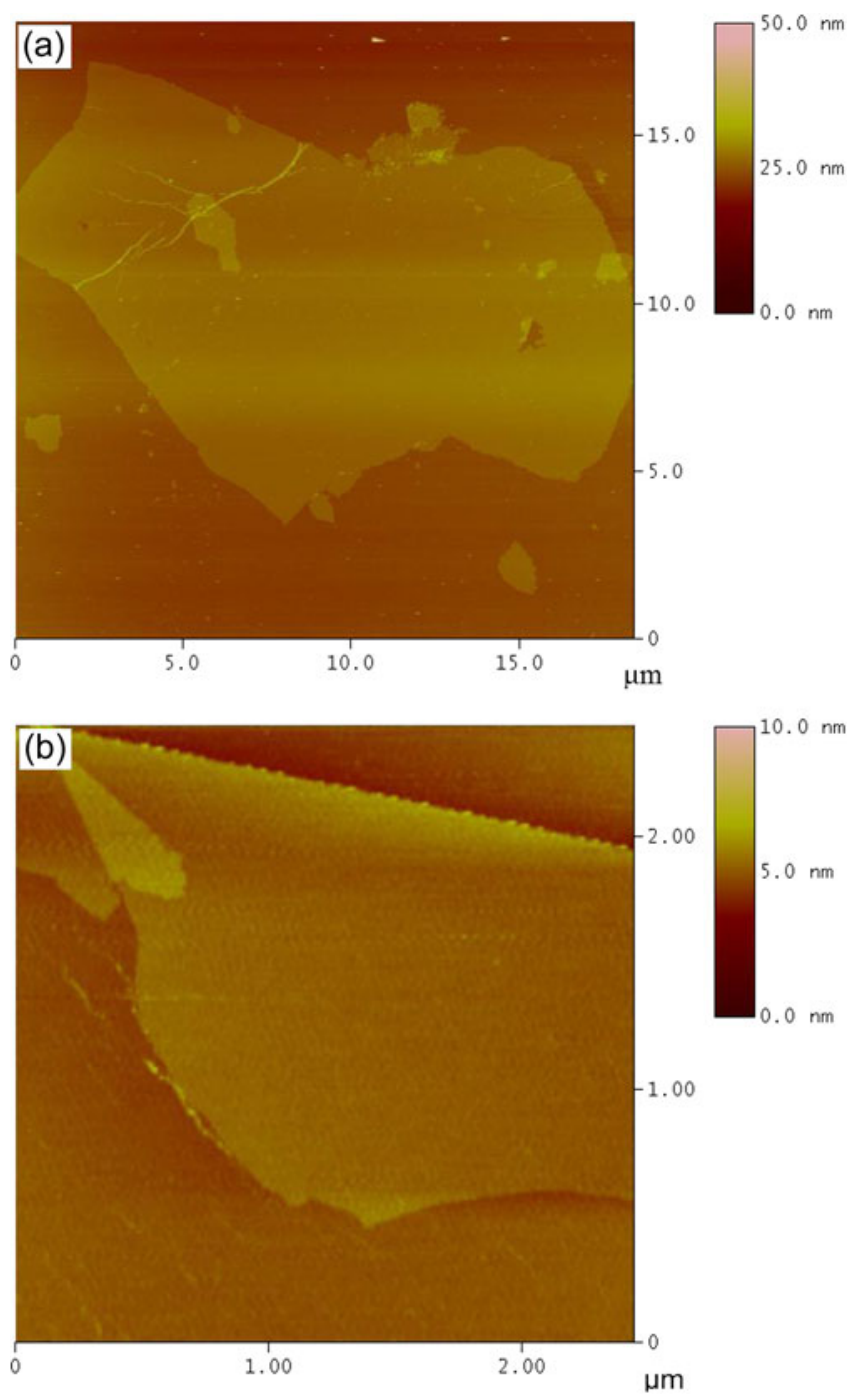

Figure 2. Tapping mode AFM images of exfoliated GO (a) and CRGO (b). Samples were prepared by drop-casting corresponding dilute dispersions onto mica sheets. 

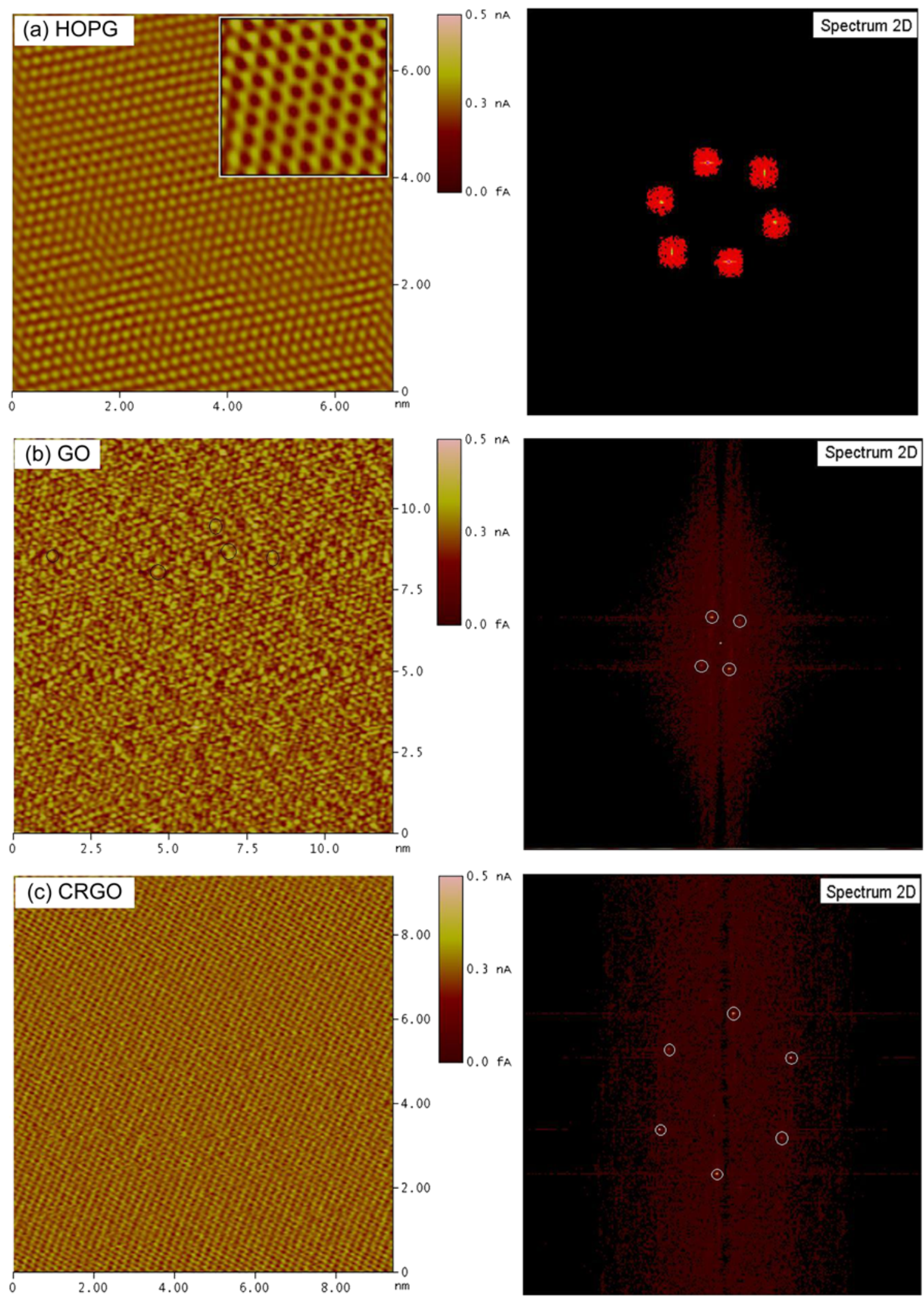

Figure 3. STM images of HOPG (a), exfoliated GO (b) and CRGO (c). Tunneling parameters: $I=0 \cdot 5 \mathrm{nA}, V=$ $50 \mathrm{mV}(\mathbf{a}) ; I=0.3 \mathrm{nA}, V=120 \mathrm{mV}$ (b) and $I=0.2 \mathrm{~A}, V=80 \mathrm{mV}$ (c). Raw STM data in left show lattice image of samples and 2D-FFT measured lattice periodicity for samples shown in right. 
respectively. Rough morphology of GO sheets is ascribed to the structural disorder induced by oxidation.

To obtain further information on the local atomic structure and to assess the quality and continuity of GO and CRGO, STM is used to image the samples which are shown in figure 3. From figure 3(a), we can see that commonly reported triangular lattice is with a bias voltage of $50 \mathrm{mV}$ (sample positive) and a tunneling current of $0.5 \mathrm{nA}$. Both triangular and honeycomb structures are simultaneously observed with the scan area decreased down to $1.5 \mathrm{~nm}$ (as insert). The peak-to-peak distance between spots of $\sim 0.24 \mathrm{~nm}$ was measured, which is very close to the theoretical value of $\sim 0.25 \mathrm{~nm}$ (Affoune et al 2001). 2-D fast Fourier transform (FFT) plot is extremely useful in removing electrical and acoustic noise from images and may also be used to isolate certain surface features (Mao et al 2004). The corresponding FFT of STM image from figure 3(a) shows a hexagonal pattern and allows a quantification of the periodicity to $0.246 \mathrm{~nm}$. Similar constant height studies were performed on an oxidized graphene sheet as shown in figure 3(b). The results reveal that parts of oxidized graphene surface have a weak triangular lattice similar to HOPG $(120 \mathrm{mV}$, $0.3 \mathrm{nA})$. As shown in figure $3(\mathrm{~b})$, it is interesting that there are many disorganized 'big' atoms around the orderly arranged $\mathrm{C}$ atoms. Apparent 'big' atoms may be $\mathrm{O}$ atoms or hydroxyl groups bonded to the top and bottom of a graphene sheet to form GO. Contrary to previous reports (Pandey et al 2008), we have not directly observed rectangular array of $\mathrm{O}$ atoms that are bound to the graphene sheet, but rectangular lattice can also be seen in FFT image. It indicated the periodic structure of graphite which is destroyed during the oxidation. For CRGO, a periodic structure similar to graphite is caught under $60 \mathrm{mV}$ and $0.3 \mathrm{nA}$. The corresponding FFT image provides evidences to show reconstruction of honeycomb structure from GO to CRGO.

Frictional properties of the samples on a mica surface were measured using FFM mode of an ambient AFM system. The tests were performed in $1 \times 1 \mu \mathrm{m}$ region with a scanning rate of $1 \mathrm{~Hz}$. The normal force $\left(F_{\mathrm{Z}}\right)$, frictional force $\left(F_{\mathrm{L}}\right)$ and friction coefficient $(\mu)$ could be calculated by the following formulas (Schwarz et al 1996):

$$
\begin{aligned}
& F_{\mathrm{Z}}=\frac{E t^{3} w}{4 L^{3} m} \Delta V_{\mathrm{V}}, \\
& F_{\mathrm{L}}=\frac{G w t^{3}}{4 L^{2} h m} \Delta V_{\mathrm{L}}, \\
& \mu=\frac{F_{\mathrm{L}}}{F_{\mathrm{Z}}}=\frac{\frac{G w t^{3}}{4 L^{2} h m} \Delta V_{\mathrm{L}}}{\frac{E t^{3} w}{4 L^{3} m} \Delta V_{\mathrm{V}}}=\left(\frac{G L}{E h}\right)\left(\frac{\Delta V_{\mathrm{L}}}{\Delta V_{\mathrm{V}}}\right),
\end{aligned}
$$

where $w, L, t, m, E$ and $G$ denote the width, length, thickness, sensitivity, Young's modulus and torsion modulus of the cantilever respectively, $h$ the height of the tip. $\Delta V_{\mathrm{L}}$ and $\Delta V_{\mathrm{V}}$ the displacement-voltage of the cantilever caused by torsion and bending respectively. Data of

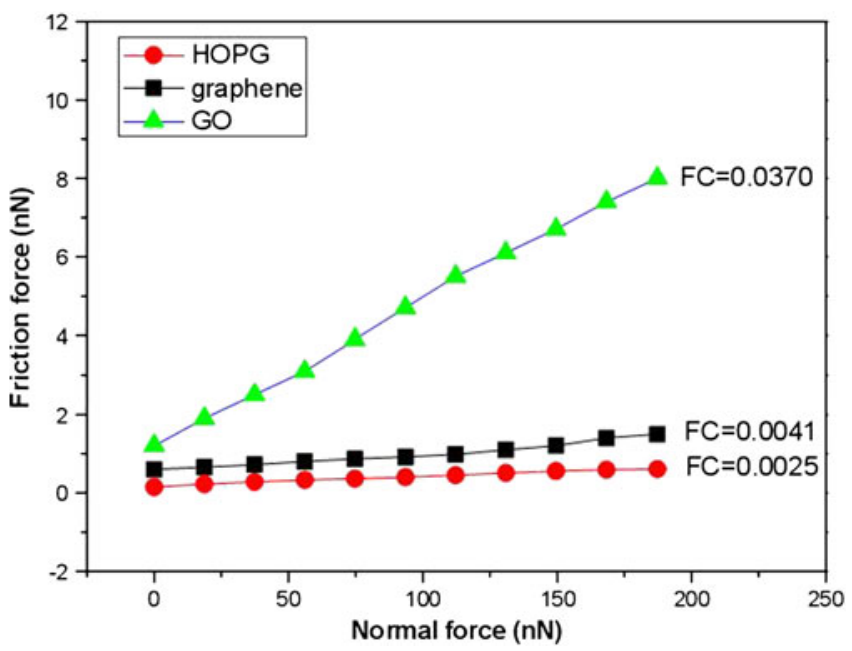

Figure 4. Normal vs average frictional force measured using same AFM tip averaged over repeated measurements on HOPG, GO and graphene.

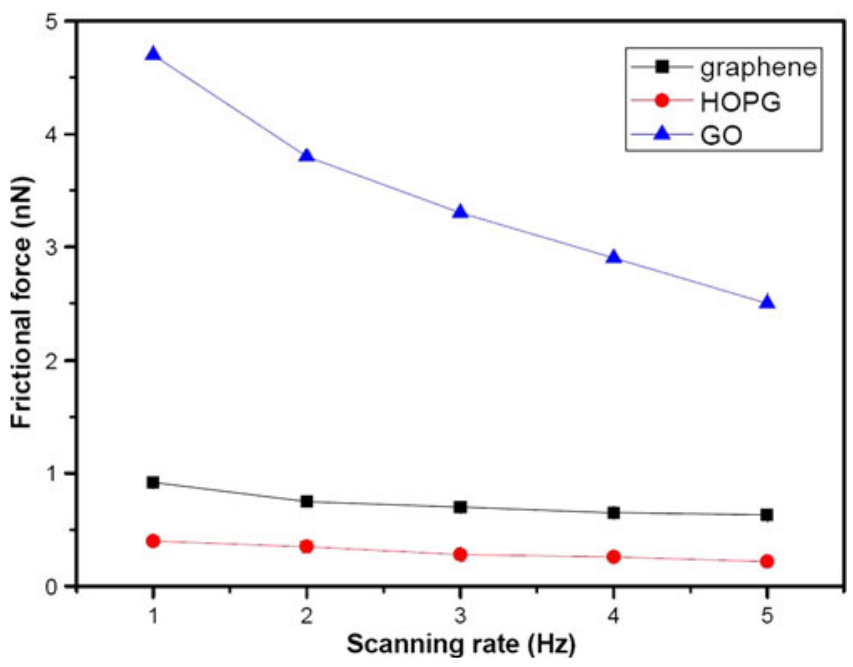

Figure 5. Effect of scanning rates on frictional forces.

the frictional force and the normal force under different setpoint were shown in figure 4 . Setpoint voltage varied from 0 to $1.0 \mathrm{~V}$, which indicated the normal force was in the range of $0-200 \mathrm{nN}$. As shown in the figure, frictional force increased along with the normal force. It is shown that the frictional force is linearly proportional to the normal force. After linear fitting, the friction coefficients of HOPG, graphene and GO were $0.0025,0.0041$ and 0.0370 , respectively. Remarkably, the frictional force was not equal to zero when the normal force applied on AFM tip was set to zero, which could be caused by the adhesion force between the tip and surface of samples (Ding et al 2011).

As shown in figure 5, the effect of scanning rate on the frictional force was also studied. During the friction, adhesion alternated with slipping between AFM tip and surface of the samples. With the increase of scanning rate, increase of kinetic energy could make it difficult for the complete 
formation of a meniscus. It indicated that the frictional force decreased along with the increase of scanning rate. Furthermore, the larger the adhesion force between the tip and the surface is, the more effect it has on the frictional force induced by the scanning rate.

\section{Conclusions}

The atomic scale structural features of single-layer GO and graphene nanosheets prepared by chemical method were studied by STM. 2D-FFT plots exhibited a reconstruction of honeycomb structure from GO to CRGO. FFM results showed that the frictional force is linearly proportional to the normal force of GO, graphene and HOPG. The friction coefficients of HOPG, graphene and GO were 0.0025, 0.0041 and 0.0370 , respectively. For all the three samples, the frictional force decreased along with the increase of scanning rate.

\section{Acknowledgements}

The financial support from the National Natural Science Foundation of China (No. 51002128), the National Science Foundation for Post-doctoral Scientists of China (No. 2012M511737) and the Undergraduate Innovation Foundation of Xiangtan University is greatly acknowledged.

\section{References}

Affoune A M, Prasad B L V, Sato H, Enoki T, Kaburagi Y and Hishiyama Y 2001 Chem. Phys. Lett. 34817

Allen M J, Tung V C and Kaner R B 2010 Chem. Rev. 110132

Ding Y, Jiang Y, Xu F, Yin J, Ren H, Zhuo Q, Long Z and Zhang P 2010 Electrochem. Commun. 1210

Ding Y, Zhang P, Ren H, Zhuo Q, Yang Z, Jiang X and Jiang Y 2011 Appl. Surf. Sci. 2581077

Dreyer D R, Park S, Bielawski C W and Ruoff R S 2010 Chem. Soc. Rev. 39228

Gass M H, Bangert U, Bleloch A L, Wang P, Nair R R and Geim A K 2008 Nature Nanotechnol. 3676

Li D and Kaner R B 2008 Science 3201170

Loh K P, Bao Q, Ang P K and Yang J 2010 J. Mater. Chem. 20 2277

Mao G, Dong W, Kurth D G and Möhwald H 2004 Nano Lett. 4 249

Muszynski R, Seger B and Kamat P V 2008 J. Phys. Chem. C 1125263

Pan B Y, Zhang H, Shi D, Sun J, Du S, Liu F and Gao H 2009 Adv. Mater. 212777

Pandey D, Reifenberger R and Piner R 2008 Surf. Sci. 6021607

Park S and Ruoff R S 2009 Nature Nanotechnol. 4217

Schwarz U D, Koster P and Wiesendanger R 1996 Rev. Sci. Instrum. 672560

Shioyama H 2001 J. Mater. Sci. Lett. 20499

Stankovich S et al 2006 Nature 442282

Stankovich S et al 2007 Carbon 451558

Wang G, Yang J, Park J, Gou X, Wang B, Liu H and Yao J 2008 J. Phys. Chem. C 1128192 